www.jmscr.igmpublication.org Impact Factor 5.244

Index Copernicus Value: 83.27 ISSN (e)-2347-176x ISSN (p) 2455-0450 crossref DOI: https://dx.doi.org/10.18535/jmscr/v5i1.08

\title{
Salvaging a Crush Avulsed Ischemic Upper Limb with Complete Radial and Ulnar Artery Transection at a Peripheral Medical Setup: Case Report
}

\author{
Author \\ Dr. A. P. Chitragupta MD \\ Department of Anaesthesiology \& Critical Care, Military Hospital Bikaner, Rajasthan India Pin 334001 \\ Email: 27chitraagupta@gmail.com
}

\begin{abstract}
s
With expanding means of transportation, and increase in outdoor and adventure motorsports activities, there has been a surge in the number of road traffic accidents and associated fatalities. The associated mortality and loss of appendages are emerging as a major public health concerns. Recent advances in surgical repair procedures and reconstruction techniques have facilitated more and more life and limb salvaging surgeries. The Mangled Extremity Severity Score (MESS) remains the most used scoring system; however it has not been validated for mangled injuries of upper extremities. Performing a temporary intravascular shunting in an attempt to salvage a precious upper limb, at a peripherally located medical setup is challenging. We present a case of severely injured left upper forearm with associated ischemia due to severed radial and ulnar artery, successfully salvaged at a sub optimally equipped peripherally located medical setup in highly uncongenial terrains of north east part of India.

Keywords: Road Traffic Accidents, MESS, Upper Extremities, Limb Salvage, Temporary Intra vascular shunting, uncongenial terrain.
\end{abstract}

\section{Introduction}

With expanding means of transportation, and increasing craze for outdoor adventure motorsports, there has been a surge in the number of road traffic accidents (RTAs) and associated fatalities. The associated mortality and loss of appendages are emerging as a major public health concerns, adding to the loss of productive life span. Recent advances in surgical repair procedures and reconstruction techniques have facilitated the surgical team to undertake more and more life and limb salvaging surgeries. ${ }^{1}$ A plethora of mangled extremity scoring systems are in vogue to objectively assess and guide in deciding whether to proceed with amputation, most of these scores are based on GustiloAnderson system of classifying the open fractures. ${ }^{2}$ The most widely used and referenced is the Mangled Extremity Severity Score (MESS), however all these scores are validated for mangled injuries of lower extremities. ${ }^{3}$ Severe upper limb injuries are unique, due to smaller muscle mass, they have less chances of developing crush syndrome; rich collaterals offer them a longer ischemia time which allows a critical time of approximately ten hours for reperfusion as compared to almost six hours for lower limb injuries, hence efforts at limb salvage should be considered at all times. ${ }^{4}$ We present a case of severely injured left upper forearm with 
associated ischemia due to severed radial and ulnar artery, attended at a peripherally located medical setup in highly uncongenial terrains of north east part of India.

\section{Case Report}

A $30 \mathrm{yr}$ old male pickup van driver, with a history of road traffic accident (RTA) was evacuated and brought to this medical echelon at $2200 \mathrm{hrs,}$ approximately an hour after the injury. On arrival the patient was under influence of liquor but was awake and alert with no loss of consciousness. Primary survey revealed, Pulse of 118 per minute, blood pressure of $90 / 50 \mathrm{mmHg}$, tongue was dry and parched, capillary return was markedly reduced, mild pallor was seen, pulse-oximeter revealed a saturation of 96 to 98 percent on room air in right hand, whereas left forearm was pulseless and pale, no radial pulsation or ulnar pulsations were appreciated. Secondary survey revealed extensively contaminated traumatic avulsion of left forearm with approximately $15 \mathrm{~cm}$ x $5 \mathrm{~cm}$ full thickness loss (Figure 1), with visible tendon injury and transected radial and ulnar vessels with fracture distal end radius. Other than last meal which he had taken only $2 \mathrm{hrs}$ back, history of any allergies, medications and past medical history were obtained which was negative. As Grade- II hemorrhagic shock was not ruled out, wide bore intravenous cannula were secured in the contralateral hand and fluid resuscitation was started with $1000 \mathrm{ml}$ of crystalloids and $500 \mathrm{ml}$ of 6 percent Hydroxyethyl starch. Blood samples were taken for urgent investigations and patient was shifted to operation room with a Primary goal to salvage the limb. Individual was taken up for surgery under ASA $\mathrm{III}_{\mathrm{E}}$ for "exploration, wound debridement and temporary intravascular shunting of both Ulnar \& Radial arteries. Keeping in view the patient's full stomach status, regional anesthesia was planned. "Supraclavicular Brachial Plexus Block (Left), was rendered after eliciting Paraesthesiae in left hand and fingers, $15 \mathrm{ml}$ each of 0.5 percent Bupivacaine and 2 percent Lignocaine diluted with normal saline to total $40 \mathrm{ml}$, was injected with intermittent aspirations negating any intravascular siting of the needle. The surgical anesthesia got established over ten minutes with completely immobile and insensate left upper limb. Thorough wound debridement and irrigation was carried out and wound was decontaminated. Wound exploration revealed a visible loss of approximately $10 \mathrm{~cm}$ of the segment of the radial artery and nearly $08 \mathrm{~cm}$ of ulnar artery (Figure 2). Surgical course was tumultuous as expected, in absence of any commercial vascular Polytetrafluoroethylene (PTFE) graft and operating microscope or binocular loupes. The struggle got compounded as the lumen of the arteries were narrow due to arterial crush and vasospasm, hence Central venous cannula could not be utilized; finally the $16 \mathrm{G}$ epidural catheter was flushed with heparin and used as an improvised temporary vascular shunt to restore the continuity and perfusion (Figure 3). The surgery lasted for approximately eight hours, with a blood loss of approximately $500 \mathrm{ml}$. Intra-operatively the hemodynamic stability was maintained with 1000 $\mathrm{ml}$ of Ringer lactate and $1500 \mathrm{ml}$ of Normal saline, injection Ceftriaxone $1 \mathrm{gm}$ intravenous and injection Tetanus toxoid $0.5 \mathrm{ml}$ intramuscularly was given as standard protocol. A slab was applied to support the fractured distal radius. Post temporary intravascular shunting restoration of perfusion was evident; there was improvement in the pallor of the hand in both the radial and ulnar vessels territories. Pulse-oximeter employed as a surrogate showed plethysmographic wave forms in all the digits. The Patient was referred to nearest higher medical echelon with a vascular surgery facility, where he underwent an extensive reconstructive surgery and definite vascular grafts were placed.

Patient returned back to our centre after four months for review with near normal hand functions. In this case regional anesthesia was carried out without any Peripheral nerve stimulator or ultrasonographic guidance, and surgical plane of anaesthesia lasted for more than 


\section{JMSCR Vol||05||Issue||01||Page 15180-15183||January}

eight hours without any supplementation. Our case turns interesting because epidural catheter used as improvised temporary vascular shunt at this medical setup helped immensely in avoiding ischemia and buying time to undergo a definitive reconstructive vascular surgery at a higher centre thereby salvaging a precious limb.

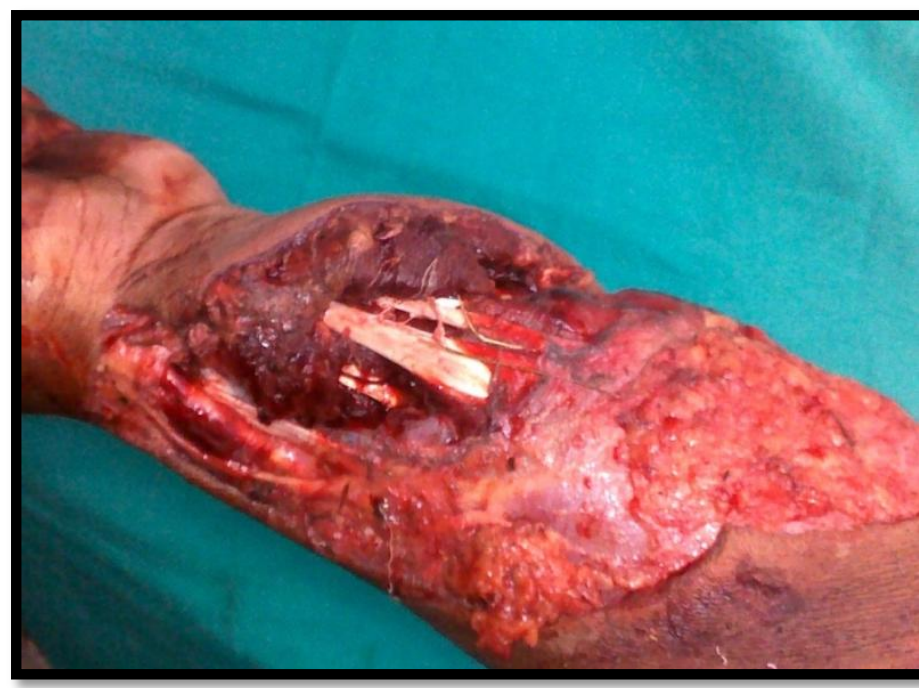

Figure 1: Extensive traumatic avulsion Left forearm approximately $15 \mathrm{~cm} \quad$ x $5 \mathrm{~cm}$ with contamination.

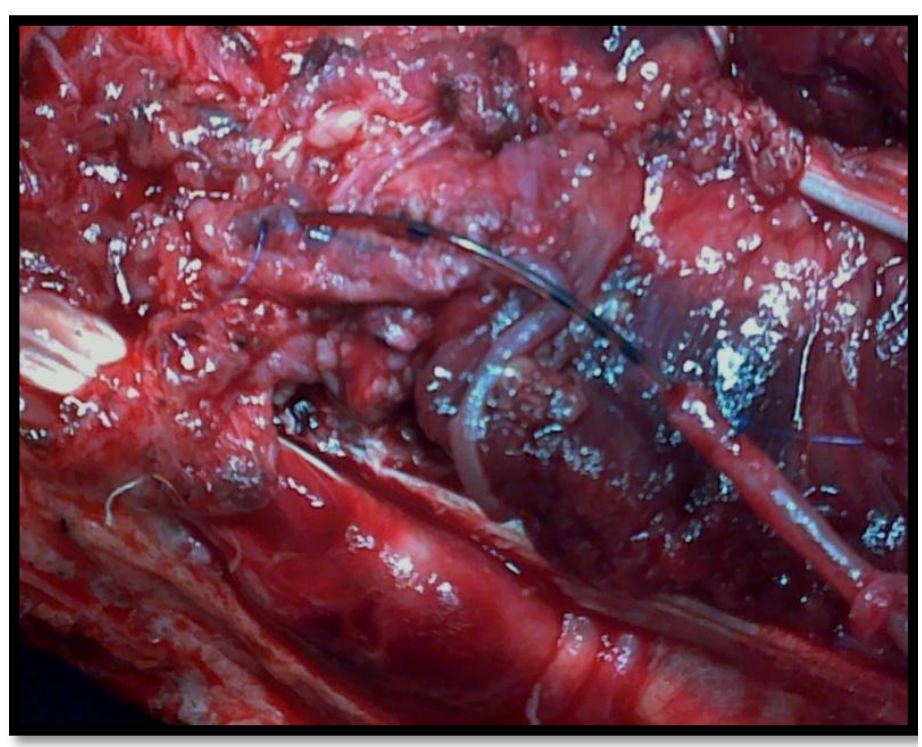

Figure 2: Visible loss of approximately $10 \mathrm{~cm}$ of the segment of the radial artery and nearly $08 \mathrm{~cm}$ of ulnar artery.

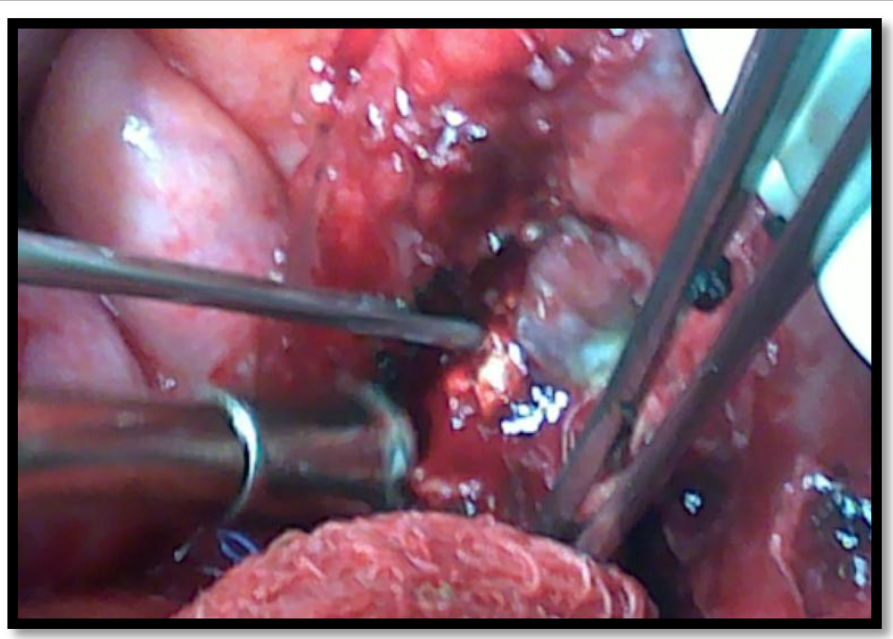

Figure 3: Heparin flushed $16 \mathrm{G}$ epidural catheter used as an improvised temporary vascular shunt to restore the continuity and perfusion.

\section{Discussion}

Mangling limb injuries are events requiring clinicians for early decision making either to amputate or salvage the limb. The MESS system of scoring was designed by Johansen et al for lower limb injuries takes in to consideration four parameters, i.e. skeletal injury, vascular injury, shock, and age and simplifies the evaluation in an emergency settings. MESS score of 7 or more has 100 percent predictive value for amputation of lower limb; however it cannot be applied as a blanket consensus in decision making for or against amputation for injuries of the upper extremities. $^{3}$

Togawa et al reported the use of MESS system for severe injuries of upper extremities, with variable outcomes and reported some successful salvage operations with a score of 7 and $11 .^{4}$ Prichayudh also reported the use of MESS in 19 patients with scores $>7$, of which 12 limbs were successfully salvaged. ${ }^{5}$ In our patient the MESS score was 8 , as evaluated by his age, transient hypotension, and pulseless extremity with poor capillary refill and very high energy trauma with contaminated soft tissue. Since this patient was full stomach and also under the influence of alcohol, regional anaesthesia was considered, keeping in view the superior quality analgesia, decreases the opioid usage, and also shorter time to regain ambulation post procedure, brachial 
plexus block was preffered. ${ }^{6}$ General endotracheal tube anaesthesia in this scenario would had necessitated a rapid sequence intubation, and subjected the patient to unnecessary airway risks and risk of aspiration.

Upper limb injuries are unique, due to smaller muscle mass, they have less chances of developing crush syndrome; rich collateral vasculature offer them a longer ischemia time allowing a critical time of approximately ten hours for reperfusion as opposed to almost six hours for lower limb injuries. Crush syndrome, combined with hemorrhagic shock, and serious infection are considered to be a definite indication for amputation.

In our case, primary survey and resuscitation went hand in hand, wound was explored, thoroughly washed and debrided in an attempt to decrease the chances of any infection, in absence of PTFE grafts, heparin flushed epidural catheter was used as a temporary vascular shunt to restore the limb perfusion. Due to terrain constraints and inability to evacuate the patient to next higher vascular centre before next 10 hours, our efforts at temporary intravascular shunting in an attempt to salvage the left upper extremity, paid off well. The patient underwent a definitive vascular repair and reconstructive surgery at the higher centre and his upper extremity was salvaged.

\section{Conclusion}

The decision to amputate or salvage a severely injured upper limb, in absence of any definite scoring system remains contentious and controversial. Restoration of vascular perfusion to the ischemic limb should be contemplated at the earliest, delay in perfusion beyond ten hours may render the limb unsalvageable, a definitive vascular reconstruction cannot be considered at a peripherally located hospital with suboptimal infrastructure, hence consideration and attempts at salvaging the upper extremity using a heparin flushed improvised temporary vascular shunt was the only viable option.

\section{References}

1. Chen ZW. Progress in limb and digital replantation: Part A-Introduction. World J Surg. 1990;14(6):804-806.

2. Gustilo RB, Mendoza RM, Williams DN. Problems in the management of type III (severe) open fractures: a new classification of type III open fractures. J Trauma 1984; 24(8): 742-6.

3. Johansen K, Daines M, Howey T “et al”. Objective criteria accurately predict amputation following lower extremity trauma. J Trauma 1990;30: 568-72.

4. Togawa S, Yamami N, Nakayama H, Mano Y, Ikegami K, Ozeki S. The validity of the mangled extremity severity score in the assessment of upper limb injuries. $\mathbf{J}$ Bone Joint Surg Br. 2005;87(11):15161519.

5. Prichayudh S, Verananvattna A, Sriussadaporn S, Sriussadaporn S, Kritayakirana K, Pak-art R. Management of upper extremity vascular injury: outcome related to the Mangled Extremity Severity Score. World J Surg.2009;33(4):857-863.

6. O'Donnell BD, Ryan H, O'Sullivan O, Iohom G.Ultrasound-guided axillary brachial plexus block with 20 milliliters local anesthetic mixture versus general anesthesia for upper limb trauma surgery: an observer-blinded, prospective, randomized, controlled trial. Anesth Analg 2009;109:279-83. 